\title{
Regional Effect on Nutritional Quality of Sorghum Hybrid Genotypes
}

\author{
U.D. Chavan", S.T. Kajjdoni, M.S. Shinde, U.S. Dalvi, S.V. Nirmal, V.R. Patil, \\ V.R. Awari, G.H. Pawar and A.S. Jadhav
}

All India Coordinated Sorghum Improvement Project, Mahatma Phule Krishi Vidyapeeth, Rahuri, Ahmednagar (MS), India

*Corresponding author

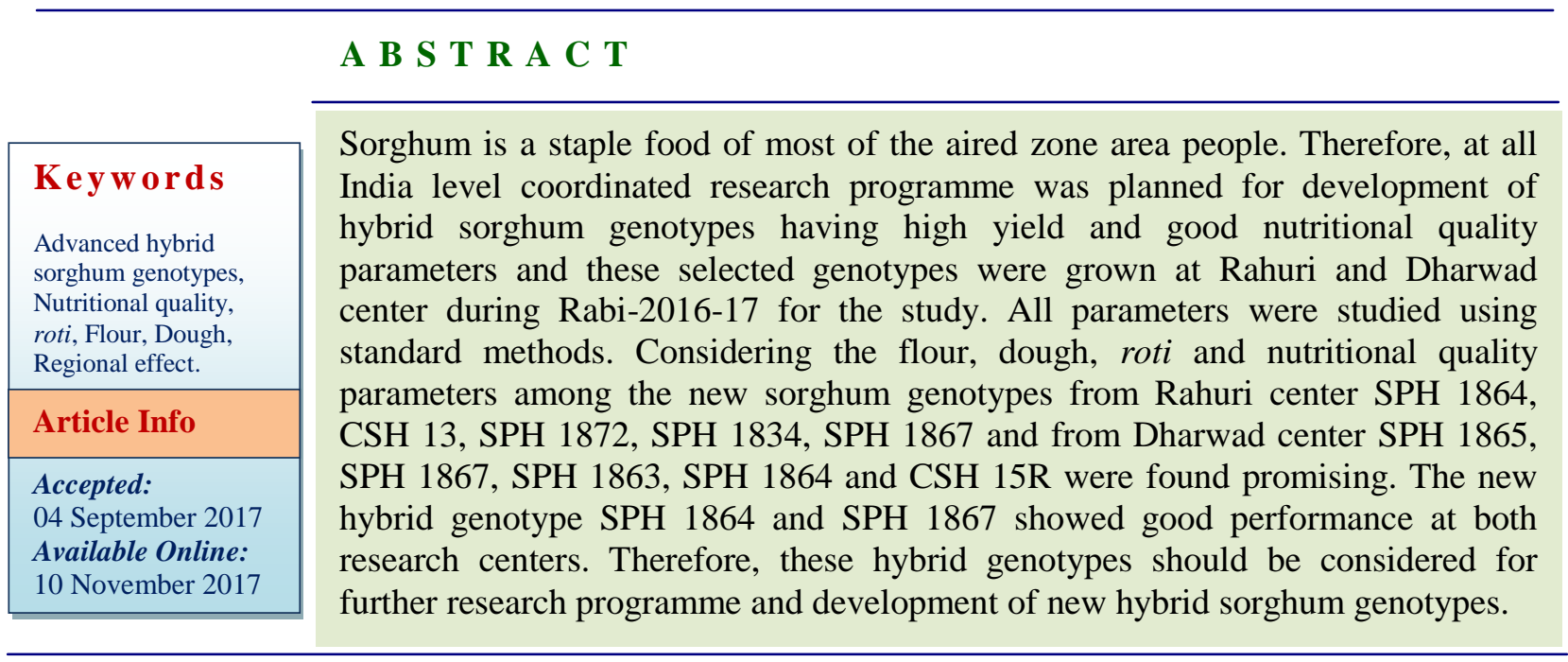

\section{Introduction}

In India sorghum is traditionally consumed in the form of unleavened pan cake/Roti/Bhakari. Because of sorghum is a staple food in many parts of the country. Though sorghum grains are nutritious, the consumption of this cereal is decreasing due to non-availability of easy cooking raw materials from the sorghum. The other major reasons are; dying traditional food habits, requirement of special skill for preparing sorghum rotis. For many years sorghum eating population particularly in rabi growing areas, the roti made from Maldandi (M 35-1) is preferred for taste and softness, over other genotypes. But now days some new genotypes of rabi sorghum are developed which gives better nutritional as well as organoleptic quality of the roti than the M 351 .

Grain sorghum [Sorghum bicolor (L.) Moench] is an important food crop particularly in arid and semi-arid tropics. It is a dual-purpose crop providing staple food for human consumption $(35 \%)$ and rest of as a fodder for livestock, alcohol production, as well as preparation of industrial products (Awika and Rooney, 2004). Many millions of people in Africa and Asia depend on sorghum as the stuff of life. Being a drought-tolerant 
crop, it can give dependable and stable yields in both kharif (rainy) and rabi (post rainy) seasons. It thrives with less rainfall than is needed for rice and maize and can be grown where no other major cereal can be grown. Altogether, sorghum is one of several really indispensable crops required for the survival of man. In India, sorghum is mainly consumed in the form of unleavened pancake (bhakri/roti). However, several indigenous processed foods such as bhatwadi, papadi, and roti are prepared and consumed in the semi-arid tropics (Rao et al., 1981; Chavan et al., 2016a, b, c). Besides, sorghum has large potential for its use in the fermentation industry, puffed products and in weaning foods for the children of developing countries. According to an FAO (2005) report, sorghum was grown globally on an area of about 46 millions ha with a production of about 60 million tons. However in India, sorghum is cultivated on an area about 9.10 million ha with a production of 7.65 million tons (Anon 2006a, b). Sorghum grains are important source of dietary proteins, carbohydrates, minerals and B group vitamins particularly to the vegetarian diets in India (Salunkhe et al., 1984; Chavan and Salunkhe, 1984; Chavan et al., 1989; Chavan and Patil, 2010; Chavan et al., 2015).

There is a considerable variation in sorghum for levels of protein, lysine, lipids, carbohydrates, fiber, calcium, phosphorus, iron, thiamine, and niacin, all these parameters imparts sorghum grain quality (Hulse, 1980; Bankar et al., 1986; Klopfenstein and Hoseney, 1995). Post rainy season (rabi) sorghum is known for its quality due to which is mostly preferred for human consumption by the masses and are characterized by lustrous, pearly white, attractive grains. Developing genotypes with high yield potential coupled with nutritionally superior quality grains is the prime objective of the breeding programme.
In India sorghum is traditionally consumed in the form of unleavened pan cake/Roti/Bhakari. Because of sorghum is a staple food in many parts of the country. Though sorghum grains are nutritious, the consumption of this cereal is decreasing due to non-availability of easy cooking raw materials from the sorghum. The other major reasons are; dying traditional food habits, requirement of special skill for preparing sorghum rotis. For many years sorghum eating population particularly in rabi growing areas, the roti made from Maldandi (M 35-1) is preferred for taste and softness, over other genotypes. But now days some new genotypes of rabi sorghum are developed which gives better nutritional as well as organoleptic quality of the roti than the M 351. This paper deals with the details of nutritional quality of hybrid grain sorghum (post rainy season) genotypes developed through a systematic breeding programme and compared with traditional local checks.

\section{Materials and Methods}

\section{Materials: Sorghum grains}

Newly developed sorghum grain samples from initial advanced hybrid trials (IAHT) were collected from all India Co-ordinated Sorghum Improvement Project, Rahuri, Maharashtra and Dharwad, Karnataka, India during Rabi-2016-17 season for flour, dough, grain, roti and nutritional quality evaluation and further prospective.

\section{Methods: Cleaning sorghum grains}

The sorghum grains were cleaned to remove all extraneous material and damaged grains.

\section{Milling of sorghum grains}

Cleaned sorghum grains were subjected to milling in laboratory grinding mill. Whole 
sorghum flour was used for nutritional quality parameters testing and preparation of roti product.

\section{Physical parameters}

The physical parameters such as hectoliter weight $(\mathrm{kg} / \mathrm{hl})$, water absorption of flour $(\%)$, Kneading quality (Scale 1-3), Spreading quality (Scale 1-3), water required for dough (\%) were estimated by standard methods of AOAC, (1990).

\section{Nutritional quality of sorghum grain}

The sorghum grain flour was then analyzed for crude protein, total sugars, soluble protein, and free amino acids and phenolics contents using standard procedure of AOAC, (1990).

\section{Preparation of sorghum roti}

The flour was made from milling grains and fine flour was made in to dough with water. The $100 \mathrm{~g}$ sorghum flour was taken for preparation of roti. The dough was well kneaded, divided into small balls, flattened on a hard wooden or metal surface sprinkled with a small quantity of flour and was baked on both sides on a hot pan (Shobha et al., 2008). The prepared rotis were then kept in bamboo basket covered with cloth piece and stored at room temperature for studying the extension of shelf life.

\section{Sensory evaluation of sorghum roti}

The sensory evaluation for different quality parameters like colour and appearance, flavour, texture, taste and overall acceptability was carried out immediately after preparation of roties at room temperature by semi trained panel of 10 judges on a 9 point hedonic scale (Amerine et al., 1980). The storage study was carried out and weight loss measured at every 4, 8, 12 and $24 \mathrm{~h}$.

\section{Statistical analysis}

All results obtained in the present study were analysed using standard methods of Panse and Sukatme (1967).

\section{Results and Discussion}

Total sixteen initial advanced hybrid sorghum genotypes were compared with local check genotype. The physical and nutritional parameters were evaluated using standard methods for judging the superiority of the hybrid genotype for further improvement in sorghum. The results on flour, dough, roti and nutritional quality are presented in Tables 14.

\section{Nutritional quality}

\section{Hectoliter weight}

The hectoliter weight gives the soundness of the grain as well as higher recovery of the flour. It is a unit weight of the grain in a specific volume. The hectoliter weight ranged from 68.30 to $78.70 \mathrm{~kg} / \mathrm{hl}$ for Rahuri centers' genotypes while 75.40 to 81.70 for Dharwad centers' genotypes respectively. The SPH 1834 hybrid genotype gave higher hectoliter weight than rest of the genotypes studied at both centers (Tables 1 and 3). Similar results are recorded by Chavan et al., (2016d, e).

\section{Water absorption capacity}

The water absorption capacity is positively correlated to the roti quality. The higher the water absorption capacity the superior was the quality of the roti due to keep the roti smooth and soft for longer time. Staling effect will be extended for longer time and it will remain fit for consumption. The water absorption capacity of flour ranged from 60 to $100 \%$ for Rahuri centers hybrid genotypes and 80 to 110 for Dharwad centers hybrid genotypes. 
Table.1 Nutritional constituents responsible for roti quality prepared from different Rabi-2016-2017 (IAHT) cultivars of sorghum (Rahuri Center)

\begin{tabular}{|c|c|c|c|c|c|c|c|c|c|c|}
\hline Genotype & $\begin{array}{l}\text { Colour } \\
\text { of the } \\
\text { grain }\end{array}$ & $\begin{array}{l}\text { Appearance/ } \\
\text { Shape of the } \\
\text { grain }\end{array}$ & $\begin{array}{c}\text { Hectoliter } \\
\text { weight } \\
(\mathrm{Kg} / \mathrm{hl})\end{array}$ & $\begin{array}{c}\text { Water } \\
\text { absorpti } \\
\text { on } \\
(\mathrm{ml} / 100 \\
\mathrm{g})\end{array}$ & $\begin{array}{l}\text { Crude } \\
\text { Protein } \\
(\%)\end{array}$ & $\begin{array}{c}\text { Soluble } \\
\text { proteins } \\
(\%)\end{array}$ & $\begin{array}{c}\text { Total } \\
\text { sugars } \\
(\%)\end{array}$ & $\begin{array}{c}\text { Starch } \\
(\%)\end{array}$ & $\begin{array}{c}\text { Free amino } \\
\text { acids } \\
(\mathrm{mg} / 100 \mathrm{~g})\end{array}$ & Phenolics (\%) \\
\hline SPH 1871 & $\mathrm{CW}$ & $\mathrm{O}$ & 76.83 & 90 & 9.72 & 1.42 & 1.71 & 51.86 & 70.53 & 2.15 \\
\hline SPH 1868 & $\mathrm{CW}$ & $\mathrm{O}$ & 76.38 & 73 & 10.50 & 2.03 & 2.12 & 55.82 & 69.03 & 2.91 \\
\hline SPH 1872 & $\mathrm{CW}$ & $\mathrm{O}$ & 72.69 & 80 & 10.10 & 1.55 & 1.63 & 42.94 & 61.16 & 2.15 \\
\hline SPH 1870 & $\mathrm{CW}$ & $\mathrm{O}$ & 75.17 & 76 & 9.40 & 1.52 & 1.64 & 50.37 & 81.57 & 2.23 \\
\hline SPH 1867 & $\mathrm{CW}$ & $\mathrm{O}$ & 77.76 & 60 & 10.98 & 1.53 & 2.08 & 45.61 & 84.73 & 2.78 \\
\hline SPH 1801 & $\mathrm{CW}$ & $\mathrm{O}$ & 78.70 & 88 & 10.60 & 1.47 & 2.00 & 46.48 & 81.46 & 2.59 \\
\hline SPH 1866 & $\mathrm{CW}$ & $\mathrm{O}$ & 77.08 & 64 & 9.18 & 1.45 & 1.78 & 52.19 & 81.57 & 2.02 \\
\hline CSH 13 & $\mathrm{CW}$ & $\mathrm{O}$ & 76.31 & 100 & 9.86 & 1.98 & 1.98 & 43.66 & 70.03 & 2.39 \\
\hline SPH 1864 & $\mathrm{CW}$ & $\mathrm{O}$ & 76.60 & 86 & 9.60 & 1.51 & 1.94 & 54.15 & 86.38 & 2.52 \\
\hline SPH 1865 & $\mathrm{CW}$ & $\mathrm{O}$ & 77.40 & 60 & 10.35 & 1.39 & 1.83 & 50.18 & 90.73 & 2.60 \\
\hline CSH 15R & CW- & $\mathrm{O}$ & 76.10 & 70 & 10.67 & 1.21 & 1.91 & 50.76 & 85.89 & 2.58 \\
\hline SPH 1863 & $\mathrm{CW}$ & $\mathrm{O}$ & 77.04 & 74 & 10.87 & 1.22 & 1.99 & 48.31 & 89.22 & 2.53 \\
\hline SPH 1869 & $\mathrm{CW}$ & $\mathrm{O}$ & 76.19 & 80 & 10.67 & 1.65 & 2.05 & 57.12 & 71.97 & 3.12 \\
\hline SPH 1837 & $\mathrm{CW}$ & $\mathrm{O}$ & 76.99 & 80 & 10.32 & 0.90 & 1.83 & 51.61 & 82.97 & 1.72 \\
\hline SPH 1834 & $\mathrm{CW}$ & $\mathrm{O}$ & 76.24 & 72 & 9.22 & 1.64 & 1.60 & 49.21 & 65.60 & 2.06 \\
\hline Phule Vasudha & $\mathrm{CW}$ & $\mathrm{O}$ & 77.51 & 74 & 10.12 & 1.75 & 2.11 & 54.55 & 71.51 & 2.50 \\
\hline Range & - & - & $\begin{array}{c}68.30- \\
78.70\end{array}$ & $60-100$ & $\begin{array}{l}9.18- \\
10.98\end{array}$ & $\begin{array}{c}0.90- \\
2.03\end{array}$ & $\begin{array}{l}1.63- \\
2.57\end{array}$ & $\begin{array}{c}42.94- \\
57.12\end{array}$ & $61.16-90.73$ & $1.50-2.91$ \\
\hline Mean & - & - & 76.08 & 77.00 & 10.02 & 1.54 & 1.93 & 50.35 & 74.83 & 2.37 \\
\hline S.E. \pm & - & - & 2.30 & 10.31 & 0.71 & 0.28 & 1.92 & 3.90 & 14.56 & 0.40 \\
\hline C.D. at $5 \%$ & - & - & 6.91 & 30.94 & 2.13 & 0.86 & 5.78 & 11.70 & 43.68 & 1.21 \\
\hline
\end{tabular}

Replications: $3 ;-$ = No sufficient seed.

Grain colour: Creamy $=\mathrm{C}$, Creamy White $=\mathrm{CW}$, Dull White $=\mathrm{DW}$, White $=\mathrm{W}$, Brown $=\mathrm{B}$, and Dull Black $=\mathrm{DB}$.

Grain Shape: Round $=\mathrm{R}$, Oval/Oblong $=\mathrm{O}$ and Wrinkle $=\mathrm{W}$. 
Table.2 Organoleptic quality of roti prepared from different hybrid/varieties of Rabi-2016-2017 (IAHT) cultivars of sorghum (Rahuri Center)

\begin{tabular}{|c|c|c|c|c|c|c|c|c|c|c|c|c|}
\hline \multirow[t]{2}{*}{ Genotype } & \multirow{2}{*}{$\begin{array}{c}\text { Water } \\
\text { required } \\
\text { for dough } \\
\quad(\mathrm{ml})\end{array}$} & \multirow[t]{2}{*}{$\begin{array}{l}\text { Kneadin } \\
\text { g quality }\end{array}$} & \multirow{2}{*}{$\begin{array}{l}\text { Spreadi } \\
\text { ng } \\
\text { quality }\end{array}$} & \multicolumn{5}{|c|}{ Organoleptic quality parameters } & \multirow{2}{*}{$\begin{array}{c}\text { Rank } \\
\text { by } \\
\text { DMRT }\end{array}$} & \multicolumn{3}{|c|}{$\begin{array}{l}\text { Loss in weight during storage } \\
\qquad(\%)\end{array}$} \\
\hline & & & & $\begin{array}{l}\text { Colour \& } \\
\text { appearanc } \\
\text { e }\end{array}$ & Texture & Flavour & Taste & $\begin{array}{c}\text { Overall } \\
\text { acceptabili } \\
\text { ty }\end{array}$ & & $4 \mathrm{hrs}$ & $8 \mathrm{hrs}$ & $24 \mathrm{hrs}$ \\
\hline SPH 1871 & 100 & 1 & 1 & 6.00 & 6.20 & 6.00 & 6.00 & 6.05 & 13 & 2.68 & 4.74 & 10.21 \\
\hline SPH 1868 & 80 & 1 & 1 & 6.20 & 6.00 & 5.80 & 6.20 & 6.05 & 13 & 2.28 & 4.81 & 10.24 \\
\hline SPH 1872 & 90 & 1 & 1 & 7.40 & 7.20 & 7.20 & 7.00 & 7.20 & 3 & 2.65 & 4.43 & 10.60 \\
\hline SPH 1870 & 85 & 1 & 1 & 6.80 & 6.40 & 6.60 & 6.80 & 6.65 & 10 & 2.35 & 4.26 & 10.86 \\
\hline SPH 1867 & 80 & 1 & 1 & 7.40 & 7.00 & 6.60 & 7.00 & 7.00 & 5 & 2.24 & 4.15 & 10.15 \\
\hline SPH 1801 & 85 & 1 & 1 & 6.20 & 6.40 & 5.80 & 6.60 & 6.25 & 12 & 2.32 & 4.10 & 10.23 \\
\hline SPH 1866 & 75 & 1 & 1 & 6.80 & 7.00 & 6.60 & 7.40 & 6.95 & 6 & 2.91 & 4.38 & 10.33 \\
\hline CSH 13 & 105 & 1 & 1 & 7.20 & 7.40 & 7.60 & 7.40 & 7.40 & 2 & 2.23 & 4.12 & 10.65 \\
\hline SPH 1864 & 90 & 1 & 1 & 7.60 & 7.60 & 7.40 & 7.60 & 7.55 & 1 & 2.15 & 3.83 & 10.57 \\
\hline SPH 1865 & 70 & 1 & 1 & 7.20 & 6.40 & 6.80 & 6.80 & 6.80 & 9 & 2.26 & 4.53 & 11.21 \\
\hline CSH 15R & 80 & 1 & 1 & 6.20 & 6.20 & 6.80 & 7.00 & 6.55 & 11 & 2.34 & 4.31 & 10.35 \\
\hline SPH 1863 & 85 & 1 & 1 & 7.00 & 6.80 & 6.80 & 6.80 & 6.85 & 8 & 2.37 & 4.46 & 10.08 \\
\hline SPH 1869 & 90 & 1 & 1 & 7.00 & 6.80 & 7.00 & 6.80 & 6.90 & 7 & 2.53 & 4.86 & 10.71 \\
\hline SPH 1837 & 90 & 1 & 1 & 7.20 & 7.40 & 6.80 & 7.00 & 7.10 & 4 & 2.43 & 4.21 & 10.67 \\
\hline SPH 1834 & 80 & 1 & 1 & 7.60 & 6.80 & 6.80 & 7.20 & 7.10 & 4 & 2.55 & 4.38 & 10.82 \\
\hline $\begin{array}{l}\text { Phule } \\
\text { Vasudha }\end{array}$ & 85 & 1 & 1 & 6.80 & 6.60 & 7.00 & 6.80 & 6.80 & 9 & 2.34 & 4.37 & 10.86 \\
\hline Range & $75-105$ & - & - & $6.00-7.60$ & $\begin{array}{c}6.00- \\
7.60\end{array}$ & $\begin{array}{l}5.80- \\
7.60\end{array}$ & $\begin{array}{c}6.00- \\
7.60\end{array}$ & $6.05-7.55$ & - & $\begin{array}{l}2.15- \\
2.91\end{array}$ & $\begin{array}{c}3.83- \\
4.86\end{array}$ & $\begin{array}{l}10.08- \\
11.21\end{array}$ \\
\hline Mean & 86 & - & - & 6.88 & 6.72 & 6.72 & 6.87 & 6.80 & - & 2.41 & 4.38 & 10.54 \\
\hline S.E. \pm & 8 & - & - & 0.50 & 0.48 & 0.48 & 0.40 & 0.42 & - & 0.18 & 0.25 & 0.30 \\
\hline C.D. at $5 \%$ & 25 & - & - & 1.51 & 1.46 & 1.44 & 1.22 & 1.27 & - & 0.57 & 0.78 & 0.90 \\
\hline
\end{tabular}

Replications: 5 minimum; - = No sufficient seed.

Kneading quality of dough, score: Good $=1$, Fair $=2$, Poor $=3$. Spreading quality of roti, score: Easy spreading without crack $=1$, Slightly difficult to spread with minute cracks $=2$, Difficult to spread with cracks $=3$.

Sensory score: Like extremely (Excellent) - 9, Like very much (Very good) - 8, Like moderately - 7, Like slightly-6, Neither like nor dislike - 5, Dislikes lightly 4, Dislike moderately - 3, Dislike very much - 2, Dislike extremely-1. 
Table.3 Nutritional constituents responsible for roti quality prepared from different Rabi-2016-2017 (IAHT) cultivars of sorghum (Dharwad Center)

\begin{tabular}{|c|c|c|c|c|c|c|c|c|c|c|}
\hline Genotype & $\begin{array}{l}\text { Colour } \\
\text { of the } \\
\text { grain }\end{array}$ & $\begin{array}{l}\text { Appearance/ } \\
\text { Shape of the } \\
\text { grain }\end{array}$ & $\begin{array}{c}\text { Hectoliter } \\
\text { weight } \\
(\mathrm{Kg} / \mathrm{hl})\end{array}$ & $\begin{array}{c}\text { Water } \\
\text { absorpti } \\
\text { on } \\
(\mathrm{ml} / 100 \\
\mathrm{g})\end{array}$ & $\begin{array}{l}\text { Crude } \\
\text { Protein } \\
(\%)\end{array}$ & $\begin{array}{c}\text { Soluble } \\
\text { proteins } \\
(\%)\end{array}$ & $\begin{array}{c}\text { Total } \\
\text { sugars } \\
(\%)\end{array}$ & $\begin{array}{c}\text { Starch } \\
(\%)\end{array}$ & $\begin{array}{c}\text { Free amino } \\
\text { acids } \\
(\mathrm{mg} / 100 \mathrm{~g})\end{array}$ & Phenolics (\%) \\
\hline SPH 1871 & $\mathrm{CW}$ & $\mathrm{O}$ & 80.57 & 85 & 9.42 & 0.82 & 2.20 & 58.20 & 81.50 & 1.07 \\
\hline SPH 1868 & $\mathrm{CW}$ & $\mathrm{O}$ & 79.55 & 85 & 9.50 & 1.03 & 2.38 & 64.59 & 71.37 & 1.34 \\
\hline SPH 1872 & $\mathrm{CW}$ & $\mathrm{O}$ & 75.40 & 90 & 9.04 & 0.66 & 1.48 & 55.47 & 68.63 & 1.00 \\
\hline SPH 1870 & $\mathrm{CW}$ & $\mathrm{O}$ & 79.30 & 80 & 8.35 & 1.17 & 2.05 & 60.68 & 79.19 & 0.93 \\
\hline SPH 1867 & $\mathrm{CW}$ & $\mathrm{O}$ & 81.04 & 100 & 11.06 & 1.05 & 2.53 & 51.07 & 79.60 & 1.58 \\
\hline SPH 1801 & $\mathrm{CW}$ & $\mathrm{O}$ & 81.22 & 90 & 9.19 & 1.21 & 2.20 & 54.44 & 78.83 & 1.46 \\
\hline SPH 1866 & $\mathrm{CW}$ & $\mathrm{O}$ & 80.71 & 90 & 9.48 & 1.15 & 2.27 & 56.61 & 82.34 & 1.54 \\
\hline CSH 13 & $\mathrm{CW}$ & $\mathrm{O}$ & 80.04 & 110 & 9.75 & 0.53 & 2.23 & 54.53 & 80.20 & 0.42 \\
\hline SPH 1864 & $\mathrm{CW}$ & $\mathrm{O}$ & 80.15 & 100 & 9.32 & 1.08 & 2.17 & 56.51 & 84.64 & 1.52 \\
\hline SPH 1865 & $\mathrm{CW}$ & $\mathrm{O}$ & 81.08 & 100 & 10.13 & 1.10 & 2.51 & 56.27 & 85.72 & 1.68 \\
\hline CSH 15R & CW- & $\mathrm{O}$ & 80.58 & 90 & 9.19 & 1.29 & 2.34 & 58.57 & 77.77 & 1.47 \\
\hline SPH 1863 & $\mathrm{CW}$ & $\mathrm{O}$ & 80.71 & 100 & 8.67 & 0.91 & 1.97 & 55.50 & 84.96 & 1.35 \\
\hline SPH 1869 & $\mathrm{CW}$ & $\mathrm{O}$ & 80.29 & 90 & 8.74 & 1.16 & 2.09 & 60.01 & 64.28 & 1.59 \\
\hline SPH 1837 & $\mathrm{CW}$ & $\mathrm{O}$ & 81.37 & 90 & 9.12 & 1.08 & 1.94 & 52.41 & 73.99 & 1.32 \\
\hline SPH 1834 & $\mathrm{CW}$ & $\mathrm{O}$ & 81.70 & 100 & 9.02 & 1.05 & 1.73 & 52.91 & 71.41 & 1.29 \\
\hline Local Check & $\mathrm{CW}$ & $\mathrm{O}$ & 79.90 & 90 & 9.16 & 1.20 & 2.35 & 65.27 & 78.11 & 1.35 \\
\hline Range & - & - & $\begin{array}{l}75.40- \\
81.70\end{array}$ & $80-110$ & $\begin{array}{l}8.35- \\
11.06\end{array}$ & $\begin{array}{c}0.53- \\
1.29\end{array}$ & $\begin{array}{l}1.48- \\
2.53\end{array}$ & $\begin{array}{l}51.07- \\
65.27\end{array}$ & $64.28-85.72$ & $0.42-1.68$ \\
\hline Mean & - & - & 80.23 & 92.81 & 9.32 & 1.03 & 2.15 & 57.07 & 77.66 & 1.31 \\
\hline S.E. \pm & - & - & 1.39 & 7.85 & 0.60 & 0.19 & 0.26 & 3.90 & 5.97 & 0.30 \\
\hline C.D. at $5 \%$ & - & - & 4.20 & 23.56 & 1.82 & 0.60 & 0.80 & 11.71 & 17.91 & 0.93 \\
\hline
\end{tabular}

Replications: $3 ;-$ = No sufficient seed.

Grain colour: Creamy $=\mathrm{C}$, Creamy White $=\mathrm{CW}$, Dull White $=\mathrm{DW}$, White $=\mathrm{W}$, Brown $=\mathrm{B}$, and Dull Black $=\mathrm{DB}$.

Grain Shape: Round $=\mathrm{R}$, Oval/Oblong $=\mathrm{O}$ and Wrinkle $=\mathrm{W}$. 
Table.4 Organoleptic quality of roti prepared from different hybrid/varieties of Rabi-2016-2017 (IAHT) cultivars of sorghum (Dharwad Center)

\begin{tabular}{|c|c|c|c|c|c|c|c|c|c|c|c|c|}
\hline \multirow[t]{2}{*}{ Genotype } & \multirow{2}{*}{$\begin{array}{c}\text { Water } \\
\text { required } \\
\text { for dough } \\
\quad(\mathrm{ml})\end{array}$} & \multirow[t]{2}{*}{$\begin{array}{l}\text { Kneadin } \\
\text { g quality }\end{array}$} & \multirow{2}{*}{$\begin{array}{l}\text { Spreadi } \\
\text { ng } \\
\text { quality }\end{array}$} & \multicolumn{5}{|c|}{ Organoleptic quality parameters } & \multirow{2}{*}{$\begin{array}{c}\text { Rank } \\
\text { by } \\
\text { DMRT }\end{array}$} & \multicolumn{3}{|c|}{$\begin{array}{l}\text { Loss in weight during storage } \\
\qquad(\%)\end{array}$} \\
\hline & & & & $\begin{array}{c}\text { Colour \& } \\
\text { appearanc } \\
\text { e }\end{array}$ & Texture & Flavour & Taste & $\begin{array}{c}\text { Overall } \\
\text { acceptabili } \\
\text { ty }\end{array}$ & & $4 \mathrm{hrs}$ & $8 \mathrm{hrs}$ & $24 \mathrm{hrs}$ \\
\hline SPH 1871 & 95 & 1 & 1 & 7.60 & 7.00 & 7.40 & 6.80 & 7.20 & 11 & 2.33 & 4.86 & 10.71 \\
\hline SPH 1868 & 98 & 1 & 1 & 7.20 & 6.80 & 7.00 & 7.20 & 7.05 & 12 & 2.13 & 4.21 & 10.67 \\
\hline SPH 1872 & 100 & 1 & 1 & 5.60 & 7.00 & 7.00 & 7.20 & 6.70 & 14 & 2.56 & 4.38 & 9.82 \\
\hline SPH 1870 & 90 & 1 & 1 & 6.40 & 6.60 & 7.00 & 7.60 & 6.90 & 13 & 2.34 & 4.37 & 10.86 \\
\hline SPH 1867 & 108 & 1 & 1 & 8.20 & 8.40 & 8.60 & 8.00 & 8.30 & 2 & 2.25 & 3.83 & 10.57 \\
\hline SPH 1801 & 100 & 1 & 1 & 7.80 & 7.60 & 8.00 & 8.00 & 7.85 & 6 & 2.26 & 4.53 & 10.31 \\
\hline SPH 1866 & 100 & 1 & 1 & 7.40 & 7.60 & 7.80 & 7.60 & 7.60 & 9 & 2.34 & 4.31 & 10.35 \\
\hline CSH 13 & 115 & 1 & 1 & 8.80 & 7.20 & 7.80 & 7.60 & 7.85 & 6 & 2.37 & 4.46 & 10.28 \\
\hline SPH 1864 & 105 & 1 & 1 & 8.60 & 7.00 & 8.40 & 8.20 & 8.05 & 4 & 2.24 & 4.15 & 10.35 \\
\hline SPH 1865 & 105 & 1 & 1 & 8.40 & 8.20 & 8.60 & 8.40 & 8.40 & 1 & 2.32 & 4.10 & 10.23 \\
\hline CSH 15R & 100 & 1 & 1 & 8.40 & 7.60 & 8.20 & 8.00 & 8.05 & 4 & 2.81 & 4.38 & 10.33 \\
\hline SPH 1863 & 110 & 1 & 1 & 8.40 & 7.80 & 8.20 & 8.00 & 8.10 & 3 & 2.23 & 4.32 & 10.45 \\
\hline SPH 1869 & 100 & 1 & 1 & 8.20 & 7.60 & 7.80 & 7.60 & 7.80 & 7 & 2.78 & 4.74 & 10.21 \\
\hline SPH 1837 & 100 & 1 & 1 & 7.60 & 7.00 & 7.80 & 7.40 & 7.45 & 10 & 2.28 & 4.61 & 10.34 \\
\hline SPH 1834 & 105 & 1 & 1 & 7.80 & 7.80 & 8.00 & 8.20 & 7.95 & 5 & 2.65 & 4.43 & 10.40 \\
\hline Local Check & 100 & 1 & 1 & 7.80 & 7.40 & 7.80 & 7.60 & 7.65 & 8 & 2.35 & 4.36 & 10.76 \\
\hline Range & $90-115$ & - & - & $5.60-8.80$ & $\begin{array}{l}6.60- \\
8.40\end{array}$ & $\begin{array}{l}7.00- \\
8.60\end{array}$ & $\begin{array}{l}6.80- \\
8.40\end{array}$ & $6.70-8.40$ & - & $\begin{array}{l}2.13- \\
2.81\end{array}$ & $\begin{array}{l}3.83- \\
4.86\end{array}$ & $\begin{array}{l}9.82- \\
10.86\end{array}$ \\
\hline Mean & 101.00 & - & - & 7.76 & 7.41 & 7.84 & 7.71 & 7.68 & - & 2.39 & 4.38 & 10.42 \\
\hline S.E. \pm & 5.00 & - & - & 0.80 & 0.48 & 0.50 & 0.41 & 0.48 & - & 0.19 & 0.23 & 0.24 \\
\hline C.D. at $5 \%$ & 17.00 & - & - & 2.42 & 1.46 & 1.52 & 1.25 & 1.45 & - & 0.58 & 0.72 & 0.74 \\
\hline
\end{tabular}

Replications: 5 minimum; - = No sufficient seed.

Kneading quality of dough, score: Good $=1$, Fair $=2$, Poor $=3$. Spreading quality of roti, score: Easy spreading without crack $=1$, Slightly difficult to spread with minute cracks $=2$, Difficult to spread with cracks $=3$.

Sensory score: Like extremely (Excellent) - 9, Like very much (Very good) - 8, Like moderately - 7, Like slightly-6, Neither like nor dislike - 5, Dislikes lightly -

4, Dislike moderately - 3, Dislike very much - 2, Dislike extremely-1. 
The hybrid genotype CSH 13 gave higher water absorption percentage than other genotypes (Table 1 and 3). These results are in agreement with previous research workers such as Michniewicz et al., (1991) and Chavan et al., (2016d, e).

\section{Crude protein}

The crude protein content ranged from 9.18\% (SPH 1866) to $10.98 \%$ (SPH 1867) in the initial advanced hybrid genotypes studied with their checks at Rahuri center. The protein content ranged from $8.35 \%$ (SPH $1870)$ to $11.06 \%$ (SPH 1867) at Dharwad center (Tables 1 and 3 ).

The genotype SPH 1867 hybrid gave highest protein content among the genotypes studied at both centers. Higher protein content is a good character for nutritional value of that genotype and also for human nutrition. These results are in agreement with previous research workers such as Michniewicz et al., (1991) and Chavan et al., (2016d, e).

\section{Soluble protein}

The soluble protein content in the flour mostly responsible for the holding more water and developing smoothness to the roti. While cooking soluble proteins, carbohydrates and free amino acids take part in the various reactions and develop specific aroma to the roti.

The soluble protein content in the flour ranged from $0.90 \%$ (SPH 1837) to $2.03 \%$ (SPH 1868) at Rahuri center trials while soluble protein ranged from 0.53 (CSH 13) to $1.29 \%(\mathrm{CSH} 15 \mathrm{R})$ at Dharwad center trials. All the genotypes were significantly different in their soluble content. These results are in agreement with previous research workers such as Michniewicz et al., (1991) and Chavan et al., (2016d, e).

\section{Total soluble sugars}

At Rahuri trials the total soluble sugars in hybrid genotypes ranged from $1.63 \%$ (SPH 1872 ) to $2.12 \%$ (SPH 1868). At Dharwad trials total sugar content ranged from 1.48 (SPH 1872) to $2.53 \%$ (SPH 1867). All the hybrid genotypes studied were significantly different. The higher sugar percentage in sorghum flour representing good amylolyptic activity while preparation of roti. Total soluble sugars are mostly responsible for good taste of the roti (Tables 1 and 3). These results are similar to Chavan et al., (2016d, e).

\section{Starch}

The starch content of the initial advanced hybrid genotypes ranged from $42.94 \%$ (SPH 1872 ) to $57.12 \%$ (SPH 1869) at Rahuri center. At Dharwad center starch ranged from $51.07 \%$ (SPH 1867) to $65.27 \%$ (Local check). Higher starch content gives good colour and amylopetic activity during roti preparation. Roti remain soft for longer time and increase self-life. Similar results are reported by Vietor et al., (1992) and Nandini and Salimath (2001).

\section{Free amino acids}

The free amino acids in the studied genotypes at Rahuri trials ranged from $61.16 \mathrm{mg} / 100 \mathrm{~g}$ flour (SPH 1872) to $90.73 \mathrm{mg} / 100 \mathrm{~g}$ flour (SPH 1865). At Dharwad center trials free amino acids ranged from $64.28 \mathrm{mg} / 100 \mathrm{~g}$ flour (SPH 1869) to $85.72 \mathrm{mg} / 100 \mathrm{~g}$ flour (SPH 1865). The initial and initial advanced hybrid sorghum genotypes were significantly different in the free amino acid content. This component mostly responsible for aroma development while roasting combines with moisture, soluble proteins and sugars. These results are in agreement with previous research workers such as Michniewicz et al., (1991) and Chavan et al., (2016d, e). 


\section{Phenolics}

The phenolics content in the studied hybrid genotypes at Rahuri center trials ranged from $1.72 \%$ (SPH 1837) to $2.91 \%$ (SPH 1868). At Dharwad trials phenolics content ranged from $0.42 \%$ (CSH 13) to $1.68 \%$ (SPH 1865). The phenolics mostly responsible for astringent taste to the product but nowadays it acts as antioxidants which prevent cancer development in human body. These nutritional quality parameters results are in agreement with Glover et al., (186), Chavan et al., (1988), Chavan et al., (2009), Chavan and Patil (2010).

\section{Roti quality}

All sorghum grain samples of initial advanced hybrid trials of Rabi-2016-17 season grown at Rahuri and Dharwad centers were used for the roti preparation and then used for organoleptic evaluation (colour and appearance, texture, falvour/aroma, taste and overall acceptability using 1 to 9 hedonic scale rating (Tables 2 and 4). On the basis of these parameters and overall acceptability Duncan Multiple Range Taste was used to give the numbering for ranking the hybrid genotypes. For smoothness of the roti storage study was also conducted and water loss (weight loss of roti) was measured at 4, 8 and $24 \mathrm{hrs}$. The results regarding weight loss in roti are presented in Table 2 and 4 . These results are in accordance with the previous research work done by Murty and Subramanian (1981), Subramanian and Jambunathan (1981; 1982), Salunkhe et al., (1984) and Shobha et al., (2008).

Considering the flour, dough, roti and nutritional quality parameters among the new hybrid sorghum genotypes from Rahuri center SPH 1864, CSH 13, SPH 1872, SPH 1834, SPH 1867 and from Dharwad center SPH 1865, SPH 1867, SPH 1863, SPH 1864 and
CSH 15R were found promising. At both research centers SPH 1864 and SPH 1867 hybrid genotypes showed good performance without any adverse effect of region. Therefore, these genotypes should be considered for further research programme and development of new hybrid sorghum genotypes.

\section{References}

Amerine, M. A., Pangborn, R. M. and Rossler, E.B. (1980). Principles of sensory evaluation of food. Academic Press, New York.

Anonymous, (2006a). Research Review Committee Meeting Report on Sorghum. Mahatma Phule Krishi Vidyapeeth, Rahuri. pp.1-182.

Anonymous, (2006b). Report on trials and nurseries kharif, rabi and forage sorghum. 36 ${ }^{\text {th }}$ Annual Group Meeting held at Marathwada Agricultural University Parbhani. 11-13 May 2006. Vol. 2: pp. 209.

AOAC, (1990). Official Methods of Analysis. $15^{\text {th }}$ Edn. Association of Official Analytical Chemists, Washington, DC, pp.113-127.

Awika, J. M. and L. W. Rooney. (2004). Sorghum phytochemicals and their potential aspects on human health. Phytochemistry. 65:1999-1221.

Bankar, J. R., Chavan, J. K. and Kadam, S. S. (1986). Effects of Incorporation of Sorghum Flour in wheat Maida on Physical and Sensory Properties of Bread, Buns, cookies and Biscuits. Journal Maharashtra Agriculture Universities 11(2): 216-218.

Chavan U. D., Jagtap Y. K., Shinde M. S and Patil J. V. (2016c). Preparation and nutritional quality of sorghum chakali. International Journal of Recent Scientific Research. 7 (1): 8404-8411. 
Chavan U. D., Pansare, S. S., Patil, J. V. and Shinde, M. S. (2015). Preparation and Nutritional Quality of Sorghum Papads. Int. J. Curr. Microbiol. App. Sci. 4(5): 806-823.

Chavan, J. K., Chavan, U. D. and Nagarkar, V. D. (1989). Effects of malting and fermentation on nutritional quality of sorghum. J. Maharashtra Agric. Univ., 14 (2) : 246-247.

Chavan, J. K., Salunkhe, D. K. (1984). Structure of sorghum grain in nutritional and processing quality of Sorghum. Qual. Plant. Pl. Foods Human Nutr 29: pp.21-31

Chavan, U. D. and Patil, J. V. (2010). Grain Processing of Sorghum. Ibdc publishers, Lucknow. pp. 10-15

Chavan, U. D., Bhagwat, V. R., Ratnavati, C. V., Patil, J. V., Gawali, H. S and Shailaja, V. (2010). Jwariche Ruchakar Padartha. Sorghum Research Centre, Rajendranagar, Hyderabad (India).pp. 26

Chavan, U. D., Jagtap, Y. K., Dalvi, U. S. and Patil, J. V. (2016b). Preparation and Nutritional Quality of Sorghum Shankarpali. Int. J. Pure App. Biosci. 4 (1): 100-108.

Chavan, U. D., Nirmal, S. V., Gadakh, S. R., Pawar, G. H. and Shinde, M. S. (2016e). Effect of location on nutritional quality of sorghum grain and roti. International Journal of Current Research, 8 (8): 36865-36869.

Chavan, U. D., Nirmal, S. V., Shinde, M. S., Pawar, G. H. and Gadakh, S. R. (2016d). Nutritional quality of advanced sorghum genotypes. International Journal of Recent Scientific Research. 7 (8): 13148-13151.

Chavan, U. D., Patil, J. V. and Shinde M. S. (2009). Nutritional and roti quality of sorghum genotypes. Indonesian Journal of Agriculture Science 10: 80-87.

Chavan, U. D., Yewale, K. V. and Dayakar
Rao, B. (2016a). Preparation of bread and cookies from sorghum flour. International Journal of Recent Scientific Research. 7 (5): 1114511153.

Chavan, U.D., Chavan, J.K. and Kadam, S.S. (1988). Effect of fermentation on soluble proteins and in-vitro protein digestibility of sorghum, green gram and sorghum + green gram blends. J. Food Sci. 53 : 1574-1575.

FAO, (2005). Food and Nutrition Series No. 27. Rome Italy.

Glover, J., Walker, C., and Mattern, P. (1986). Functionality of sorghum flour components in a high ratio cake. Journal of Food Science. 51: 1280-1283, 1292.

Hulse, J. H., E. M. Laing and O. E. Pearson. (1980). Sorghum and Millets: Their composition and nutritive value. IDRC, Ottawa, Canada: Academic Press. Pp. 977.

Klopfenstein, C. F. and Hoseney, R. C. (1995). Nutritional properties of sorghum and millets. Dendy, D. A. V 125 St Paul, Minn.: American Association of Cereal Chemistry.

Michniewicz, J., Biliaderis, C. G. and Bushuk, W. (1991). Effect of added pentosans on some physical and technological characteristics of dough and gluten. Cereal Chemistry 68: 252258.

Murty D. S. and Subramanian V. (1981). Sorghum Roti: I. Traditional Methods of Consumption and Standard Procedures for Evaluation. Proceedings of the International Symposium on Sorghum Grain Quality: 73-78.

Nandini C. D. and Salimath, P. V. (2001). Structural features of arabinoxylans from sorghum having good roti-making quality. Food Chemistry 74: 417 - 422.

Panse, V. G. and Sukhatme, P. V. (1967). Statistical Methods for Agricultural Workers $2^{\text {nd }}$ Edn. I.C.A.R., New Delhi. 
Rao Prasada, K. E. and Murty, D. S. (1981). Sorghum for Special Uses. Proceedings of the International Symposium on Sorghum Grain Quality: 129-134.

Salunkhe D. K., J. K. Chavan and S. J. Jadhav. (1984). Nutritional and processing quality of sorghum. Oxford and IBH Publishing Co, New Delhi Pp. 275.

Shobha, V., Kasturiba, B., Naik, R. K. and Yenagi, N. (2008). Nutritive Value and Quality Characteristics of Sorghum Genotypes. Karnataka Journal of Agriculture Science 20: 586-588

Subramanian, V. and Jambhunathan, R. (1982). Properties of sorghum grain and their relationship to rot quality. In: International Symposium on sorghum grain quality. ICRISAT, Patancheru, India. pp. 280-288.

Subramanian, V. and Jambunathan, R. (1981). Properties of Sorghum Grain and their Relationship to Roti Quality. Proceedings of the International Symposium on Sorghum Grain Quality: 286-288.

Vietor, R. J., Angelino, S. A. G. F. and Voragen, A. G. J. (1992). Structural features of arabinoxylans from barley and malt cell wall material. Journal of Cereal Science 15: 213-222.

\section{How to cite this article:}

Chavan, U.D., S.T. Kajjdoni, M.S. Shinde, U.S. Dalvi, S.V. Nirmal, V.R. Patil, V.R. Awari, G.H. Pawar and Jadhav, A.S. 2017. Regional Effect on Nutritional Quality of Sorghum Hybrid Genotypes. Int.J.Curr.Microbiol.App.Sci. 6(11): 75-85.

doi: https://doi.org/10.20546/ijcmas.2017.611.010 